\title{
A Fabry-Perot study of Wolf-Rayet ring nebulae in M 33
}

\author{
Anthony P. Marston \\ Physics \& Astronomy Department, Drake University, \\ Des Moines, IA 50311, USA \\ Laurent Drissen and Steve Godbout \\ Département de physique, Université Laval, Quebec, Canada
}

\begin{abstract}
We present a kinematical study of ring nebulae surrounding WolfRayet stars in the Local Group galaxy M 33, based on Fabry-Perot data obtained at the CFHT. Most of these rings show evidence for multiple phases of mass ejection from the central star. Variations are noted in the [OIII] $/ \mathrm{H} \alpha$ emission-line ratios across several ring nebulae generally associated a variation in kinematics. Significant [OIII] emission appears to arise from the collision between the fast WR wind and surrounding ejecta from a prior phase, such as a RSG or LBV.
\end{abstract}

\section{Introduction}

Observations of ring nebulae around Wolf-Rayet stars in external galaxies provide an overview of the large-scale structures formed around these stars during their multiple phases of evolution (see Marston 1995). WR stars in M 33 have previsouly been identified and ring nebula structures noted (Drissen et al. 1991). In this poster we consider the detailed structure and kinematics for several of the WR ring nebulae in M 33 that show multiple ring structures. We show that significant collisional excitation occurs in the interior regions of ring nebulae, associated with fast-moving swept-up shells, produced by the WR winds as they interact with previously ejected materials.

\section{Observations}

Observations of WR ring nebulae in M 33 were made at the CFHT using the multi-object spectro-imager in Fabry-Perot mode (MOSFP). Our observations provided both [OIII] and $\mathrm{H} \alpha$ narrow-band imaging and $\mathrm{H} \alpha$ kinematics for five ring nebulae displaying multiple concentric shells (see Fig. 1).

\section{Discussion}

Our data show that high-speed nebula-expansion around WR stars is formed only in regions of high [OIII] $/ \mathrm{H} \alpha$ ratios. These are found in regions typically only a few parsecs across at the centers of multiple ring structures (e.g., around MC 27 and MC 46). Esteban et al. (1994) have shown that other ring nebulae 


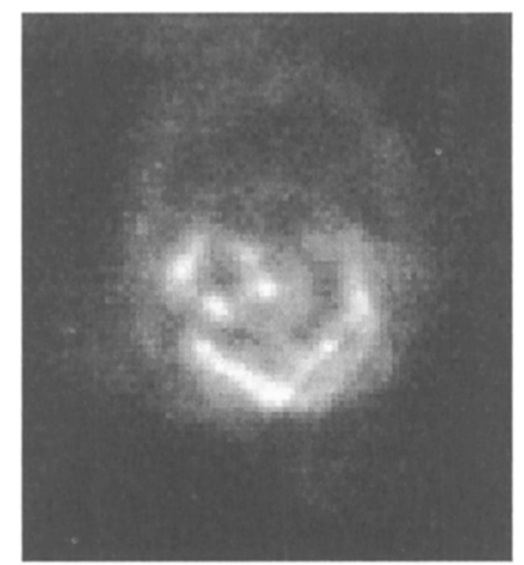

Figure 1. The multiple ring nebula around $\mathrm{MC} 27$, seen in $\mathrm{H} \alpha$. The outer shell is approximately $35 \mathrm{pc}$ across.

in $\mathrm{M} 33$ have [OIII] $/ \mathrm{H} \alpha$ ratios of between 8 and 21 , but it is noted that these cases only occur in association with large expansion velocities. Larger shells (up to $100 \mathrm{pc}$ across) show little or no expansion and low [OIII]/H $\alpha$ ratios. These larger structures have abundances similar to the surrounding ISM (Esteban et al. 1994). We suggest that the larger shells are the remnant of the early $O$ star phase of the WR stars, in which large shells of swept-up ISM are formed by the winds from the massive $\mathrm{O}$ star, which creates a cavity in the interstellar into which ejecta material, from a RSG or LBV phase of the star, flows. While the high $[\mathrm{OIII}] / \mathrm{H} \alpha$ ratio regions are caused by collisional excitation in the inner portion of multiple ring nebulae, where the fast WR winds are striking ejecta from earlier periods of WR evolution (e.g., RSG or LBV). The estimated timescales for the formation of the high-excitation regions is of order $10^{5} \mathrm{yr}$.

Acknowledgments. The work in this presentation was funded in part by a grant from NASA administered by the AAS and NASA ADP grants NAG5-2999 and NAG5-6854.

\section{References}

Drissen, L., Shara, M.M., Moffat, A.F.J. 1991, AJ 101, 1659

Esteban, C., Vílchez, J.M., Smith, L.J. 1994, AJ 107, 1041

Marston, A.P. 1995, AJ 109, 1839 